\title{
Reclutamiento 2.0
}

\section{Recruitment 2.0}

\section{Monserrat Pérez García ${ }^{1}$, Verónica Nayeli Morales García ${ }^{2}$,}

\begin{abstract}
|
The recruitment and selection of personnel is in constant evolution and we must consider using technology (ICT) and social networks to have a competitive and remarkable advantage.

The companies have gone through a series of problems in their different areas, especially the Human Resources department, which generates certain costs during the recruitment and selection process, unnecessary costs for the organization due to poor personnel selection and misuse of time, because in many occasions people confirm their attendance at job interviews or to show up to work and when the date and time are not reached, making all the process achieved with that person is time and money lost both for the company and for recruiters, this is where recruitment 2.0 should be applied.
\end{abstract}

\section{Keywords:}

Recruitment, Selection, Human Resources, Technology, Social Networks, Time.

\section{Resumen}

El reclutamiento y selección de personal está en una constante evolución y debemos considerar hacer uso de la tecnología (TICS) y redes sociales, para tener una ventaja competitiva y notable.

Las empresas han pasado por una serie de problemáticas en sus diferentes áreas, en especial el departamento de Recursos Humanos, el cual genera ciertos costos durante el proceso de reclutamiento y selección, costos innecesarios para la organización por la mala elección de personal y el mal uso de tiempo, pues en muchas ocasiones las personas confirman su asistencia a entrevistas de trabajo o a presentarse a laborar y cuando se llega la fecha y hora no llegan, hacienda que todo el proceso logrado con esa persona sea tiempo y dinero perdido tanto para la empresa como para los reclutadores, es aquí donde se debe aplicar el reclutamiento 2.0

\section{Palabras Clave:}

Reclutamiento, Selección, Recursos Humanos, Tecnología, Redes Sociales, Tiempo.

\footnotetext{
1 Estudiante de 8vo. Semestre de la Lic. Administración, Universidad Autónoma del Estado de Hidalgo, Escuela Superior Tepeji. pe276374@uaeh.edu.mx

${ }^{2}$ Estudiante de 8vo. Semestre de la Lic. Administración, Universidad Autónoma del Estado de Hidalgo, Escuela Superior Tepeji.
} 


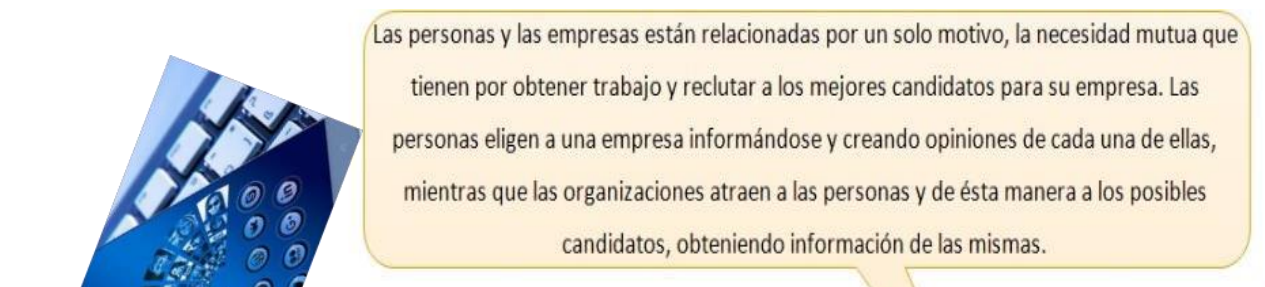
candidatos, obteniendo información de las mismas.

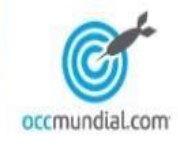

El reclutamiento es un conjunto de procedimientos orientado a atraer candidatos

potencialmente calificados y capaces de ocupar cargos dentro de la organización. El

reclutamiento debe atraer una cantidad de candidatos suficiente para abastecer de modo adecuado el proceso de selección. Además, la función del reclutamiento es suministrar la selección de materia prima básica (candidatos) para su funcionamiento. (CHIAVENATO, 2018)

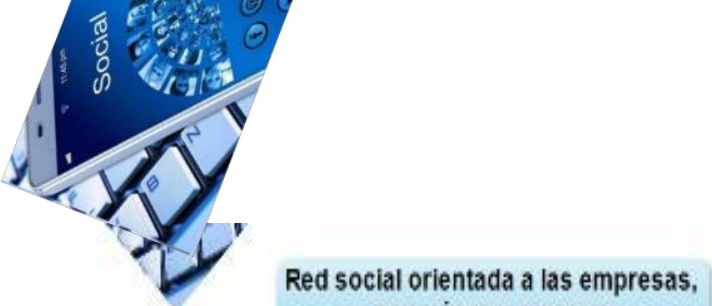
negocios y personas

Bolsa de trabajo de las mejores empresas, empleos, vacantes y ofertas de empleo.

Es un sitio web que te permite iniciar en el mundo de los trabajos por internet

Compañia estadunidense que ofrece servicio de redes sociales y medios sociales en línea

lo cual sirve para promover vacantes dentro de los network.

Motor de búsqueda similar a sitios como Google, el cual proporciona

enlaces a empleos en toda la web

\section{$\checkmark$}

Proceso de reclutamiento

Linkedin

(c) Mundial OCC Mundial

\section{CompuTrabajo}

\section{Facebook}

Twitter

Indeed

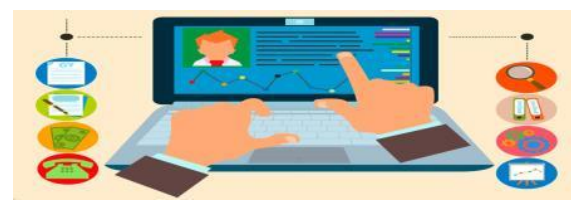

\section{formas de reclutar \\ Perifoneo \\ Volantes \\ Lonas \\ Formas de reclutar \\ Redes sociales, Blog \\ Internet}

\section{indeed}

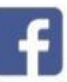

Compuirabajo portaldelempleo
Reclutamiento 2.0 es una alternativa especialmente atractiva para las pequeñas $y$ medianas empresas en términos de coste, y para nadie es un secreto que la búsqueda de candidatos a través de las redes sociales está equilibrando la balanza entre las grandes empresas y las PYMES, sobre todo en términos de costes de reclutamiento y selección, cuando se busca a los mejores candidatos. (Rojas, 2010) 


\section{Referencias}

(2014). Obtenido de Gimeno:

https://repositorio.unican.es/xmlui/bitstrea

$\mathrm{m} /$ handle/10902/9681/SAIZBRINGASCLAUDI

A.pdf?sequence $=1$

Accióntrabajo. (12 de Noviembre de 2018).

Obtenido de https://acciontrabajo.com.mx/

B, W., Jr., W., \& Keith, D. (2008).

Administracion de los recursos humanos.

México: McGraw Hill.

B., M. s. (2018). Técnicas de Reclutamiento y selección de personal. Obtenido de https://books.google.com.mx/books?id=6kW BAAAQBAJ\&pg=PA7\&dq=que+es+el+tiempo+ en+reclutamiento\&hl=es\&sa=X\&ved=0ahUK Ewjh1cfPgMDeAhVEG6wKHZMmBcQQ6AEIK TAA\#v=onepage \&q=que\%20es\%20el\%20tie $\mathrm{m}$ po\%20en\%20reclutamiento $\& \mathrm{f}=$ false

Bringas, C. S. (26 de Agosto de 2016).

Reclutamiento 2.0. Obtenido de https://repositorio.unican.es/xmlui/bitstrea m/handle/10902/9681/SAIZBRINGASCLAUDI A.pdf?sequence $=1$

Bringas, C. S. (26 de Agosto de 2016).

Reclutamiento 2.0. Obtenido de https://repositorio.unican.es/xmlui/bitstrea $\mathrm{m} /$ handle/10902/9681/SAIZBRINGASCLAUDI A. pdf?sequence $=1$

CHIAVENATO. (24 de 10 de 2018).

CHIAVENATO. Obtenido de

http://www.utntyh.com/wp-

content/uploads/2009/09/RESUMEN-

UNIDAD-5-CHIAVENATO.pdf

Chiavenato, I. (2007). Administración de

Recursos Humanos: El capital humano de las organizaciones. McGraw-Hill

Interamericana.

Colegio Oficial de León. (24 de 10 de 2018).

Obtenido de http://www.trabajosocialleon.org/perfilprofe sional.php

Concepto de. (1 de 2018). Obtenido de https://concepto.de/reclutamiento/\#ixzz5Ut $43 \mathrm{~cd} 60$

Empleo MX.com. (12 de Noviembre de 2018). Obtenido de

http://www.empleomx.com/empresas/que_ es.php

Indeed. (12 de Noviembre de 2018).

Obtenido de https://www.indeed.com.mx/

Ltd, J. S. (2018). Jora México. Obtenido de https://mx.jora.com/cms/quienes-somos

Monografias.com. (2012). Obtenido de https://www.monografias.com/trabajos38/a administración-tiempo/administracióntiempo.shtml

NominaPRO. (22 de 10 de 2018). Obtenido de https://www.nominapro.mx/blog/reclutamie nto-2-0/

Perfil laboral. (Noviembre de 2018). Obtenido de

http://www.ara.mil.ar/archivos/Docs/Perfil\% 20Profesional.pdf 